Leading Article

\title{
Treatment options for colorectal liver metastases
}

\author{
Irving Taylor
}

University Surgical Unit, University of Southampton, Southampton General Hospital, Tremona Road,
Southampton SO9 $4 X Y$, UK

As a consequence of resurgence of interest and a significant increase in active intervention for patients with colorectal liver metastases, numerous policies for such patients are available, and many clinicians are confused by the plethora of therapeutic permutations and combinations. Colorectal cancer is common and the most frequent site of recurrence (and subsequent mortality) is the liver. It is important however to distinguish between established policies and experimental treatments undergoing Phase I-II evaluations. This article reviews available management options.

\section{Screening for liver metastases}

This has been suggested on the basis that the recognition of an operable solitary liver metastasis might enable resection to occur and survival benefit to accrue. Unfortunately this argument is spurious. It is by no means certain that asymptomatic diagnosis enhances survival, because of the effect of lead-time bias. Only a randomized trial can show this. A trial investigating the role of carcinoembryonic antigen (CEA) is in progress and the results are awaited with interest. Many groups carry out regular post-operative ultrasound, and even computed tomographic (CT) scans of the liver but this practice is not universal. Usually the time-lapse between liver metastases forming and symptoms developing is short. For the majority of patients following surgery for colorectal cancer regular screening for asymptomatic metastases is probably not justified, but when symptoms such as pain and weight loss do develop, then a 'metastatic' screen is required.

Correspondence: Professor I. Taylor, M.D., Ch.M., F.R.C.S.

Received: 10 January 1992

\section{Can they be prevented?}

Surgical technique might be important. Early ligation of mesenteric vessels, careful lymphatic clearance and adequate cytotoxic washes have all been advocated. Recent encouraging reports of adjuvant therapy hold out some promise. Improved disease-free survival and overall survival for patients with Dukes' C disease with 5-fluorouracil, (5-FU) and levamisole has been demonstrated in several studies. ${ }^{1,2}$ The role of adjuvant portal vein infusion has demonstrated possible benefit ${ }^{3,4}$ but is still the subject of large randomized trials (such as the AXIS Study in the UK). It is becoming increasingly necessary to consider some form of adjuvant therapy for patients with a high risk of developing recurrent disease following surgery for colorectal cancer. However if surgeons feel that uncertainty still exists in this regard then they should consider randomizing patients into appropriate clinical trials.

\section{Pre-operative diagnosis}

Patients should undergo ultrasound scanning of liver prior to resection of colorectal cancer. This will enable sensible planning - if liver metastases are reported then $\mathrm{CT}$ scan can more accurately localize the lesion(s) and determine percentage hepatic replacement (PHR). If a solitary metastasis is present which requires major resection this should be considered, probably at a separate procedure some 6-8 weeks after colorectal resection. If a small metastasis is present on the free edge of the liver, synchronous wedge excision is justified.

Multiple metastases (up to five) in one lobe might be suitable for hepatectomy and this is advocated by several recent studies, although the evidence for benefit is based on comparison with retrospective 'historical' controls and is subject to bias. Nevertheless in order for rational decisions on treatment to be made pre-operative liver scan is necessary and should be available. 


\section{Indications for liver resection}

This is still controversial; however, solitary metastases and indeed several metastases in one lobe are suitable for resection. The patient should be fit with no evidence of recurrent disease elsewhere. This requires pre-operative CT scans of pelvis and liver (with emphasis on portal glands) as well as hepatic artery angiography to clarify the arterial anatomy prior to resection.

Solitary metastases are rare but following resection consistent reports of 3 year survivals in excess of $50 \%$ and 5 year survival of $25 \%$ are available. On recurrence however almost half the patients have disease within the remaining liver and half recurrence elsewhere. ${ }^{7}$ The argument for 'adjuvant' treatment following liver resection is strong and randomized trials indicated.

\section{Loco-regional treatment}

Most studies indicate that locoregional delivery of cytotoxic chemotherapy (via the hepatic artery) results in a better response rate than systemic delivery, with fewer side effects. ${ }^{8}$ Of the various devices available the insertion of a catheter into the gastro-duodenal artery with a subcutaneous 'port' is the most popular. Of the available regimes available, 5-fluorouracil is still favoured and its action may be enhanced by degradable starch microspheres. ${ }^{9}$ Monthly treatment is advocated with a 'flush' of the port between treatments. The side effects of this treatment are minimal.

Other possibilities include 5-fluorodeoxyuridine (but with the risk of more toxicity, especially to liver $)^{10}$ and encapsulated drugs such as adriamycin or mitomycin C. It is possible that angiotensin II might improve uptake of these drugs by tumour. ${ }^{11}$ Internal radiotherapy is still being assessed but promising data with yttrium-90 and iodine-131 lipiodol is beginning to emerge. In addition intrahepatic 5-FU and intravenous leucovorin have theoretical possibilities. The majority of these novel locoregional approaches are still being assessed in Phase I and II studies but hopefully
Phase III trials will become available in the near future.

\section{Systemic treatment}

Systemic treatment is most frequently considered in patients unsuitable for alternative treatments described above. Numerous regimens, either single agent or combination chemotherapy are available. Most have 5-FU as a 'base' and the generally quoted response rate for this drug is $20 \%$. Recently modulation of the action of 5-FU with leucovorin (folinic acid) has been demonstrated, and many studies have indicated that this combination is more effective than 5-FU alone. ${ }^{12}$ This combination (5-FU plus leucovorin) should now be considered as the first line systemic approach in advanced colorectal cancer. Some evidence exists to indicate that further benefit can accrue by addition of interferon, ${ }^{13}$ and Phase III trials are now available comparing 5-FU/leucovorin with 5-FU/leucovorin/interferon.

\section{Conclusions}

The following approaches are advocated. (1) Pre- $\overrightarrow{0} \overrightarrow{0}$ operative liver scan prior to resection of colorecta cancer. (2) Until evidence to the contrary a 'metas $\overline{0}$. tatic screen' is suggested only for suspicious symp-? toms following colorectal cancer surgery. (3) Solitary liver metastases (requiring hepatectomy) should be considered for resection as a separate procedure following full investigation. Small easily accessible metastases can be dealt with synchronously. (4) Multiple metastases in one lobe should be considered for resection provided there is no disease elsewhere. (5) Multiple liver metastases in both lobes (less than $50 \%$ ), with no disease elsewhere should be considered for locoregional treatment. (6) Multiple liver metastases (more than $50 \%$ ) with or without obvious disease elsewhere may not be suitable for any specific treatment but if systemic approaches are considered 5-FU and leucovorin are the best available.

\section{References}

1. Laurie, J.A., Moertel, C.G., Fleming, T.R. et al. Surgical adjuvant therapy of large bowel carcinoma; an evaluation of levamisole and the combination of levamisole and flourouracil. J Clin Oncol 1989, 7: 1447-1456.

2. Moertel, C.G., Fleming, T.R., McDonald, J.S. et al. Levamisole and fluorouracil for adjuvant therapy of resected colon carcinoma. $N$ Engl J Med 1990, 322: 352-358.

3. Wereldsma, J.C.J., Bruggink, E.D.M., Meijer, W.S. et al. Adjuvant portal vein liver infusion in colorectal cancer with 5-fluorouracil/heparin versus urokinase versus control. Cancer 1990, 65: 425-432.

4. Wolmark, N., Rockette, H., Wickerham, D.L. et al. Adjuvant therapy of Dukes' AC and C adenocarcinoma of the colon with portal vein 5-fluorouracil hepatic infusion. J Clin Oncol 1990, 8: 1466-1475.

5. Scheele, J., Stangl, R. \& Altendorf-Hofman, A. Hepatic metastases from colorectal carcinoma; impact of surgical resection on the natural history. $B r J$ Surg 1990, 77: 1241-1246.

6. Hunt, T.M., Carty, N. \& Johnson, C.D. Resection of liver metastases from a colorectal carcinoma does not benefit the patients. Ann R Coll Surg Engl 1990, 72: 199-205. 
7. Schlag, P., Hohenberger, P. \& Herfarth, Ch. Resection of liver metastases in colorectal cancer-competitive analysis of treatment results in synchronous versus metachronous metastases. Eur J Surg Oncol 1990, 16: 360-365.

8. Safi, E., Bittner, R., Roscher, R. et al. Regional chemotherapy for hepatic metastases of colorectal carcinoma (continuous intraarterial versus continuous intraarterial/ intravenous therapy). Cancer 1989, 64: 379-387.

9. Hunt, T.M., Flowerdew, A.D.S., Birch, S.J. et al. Prospective randomised controlled trial of hepatic arterial embolization or infusion chemotherapy with 5-fluorouracil and degradable starch microspheres for colorectal liver metastases. Br J Surg 1990, 77: 779-782.

10. Chang, A.E., Shneider, P.D., Sugarbaker, P.H. et al. A prospective randomized trial of regional versus systemic continuous 5-fluorodeoxyuridine chemotherapy in the treatment of colorectal metastases. Ann Surg 1987, 206: 685-693.
11. Goldberg, J.A., Thompson, J.A.K., Fenner, J. et al. Angiotensin II as a potential method of targeting cytotoxic-loaded microspheres in patients with colorectal liver metastases. Br J Cancer 1991, 64: 114-119.

12. Petrelli, N., Douglass, H.O., Herrera, C. et al. The modulation of fluorouracil with leucovorin in metastatic colorectal carcinoma: a prospective randomized phase III trial. J Clin Oncol 1989, 7: 1419-1426.

13. Wadler, S., Schwartz, E.L., Goldman, M. et al. Fluorouracil and recombinant alpha-2d-interferon: an active regimen against advanced colorectal carcinoma. J Clin Oncol 1989, 7: 1769-1775. 